\title{
Effect of Intermittent Warming Treatments on the Storability of Balady Mandarin (Citrus reticulata L.) Fruits
}

\author{
Mansour, A.H.A. ${ }^{2}$; A.M. El-Salhy ${ }^{1}$; F.M.A. Mostafa $^{1}$ and Samia G.E. Elorabi ${ }^{2}$ \\ ${ }^{1}$ Pomology Dept., Fac. Agric., Assiut University, Assiut, Egypt. \\ ${ }^{2}$ Agricultural Research Centre, Horticultural Research Institute - Giza.
}

\begin{abstract}
:
This study was conducted during three successive seasons 2012, 2013 and 2014 to study the effect of intermittent warming (IW) on the storability and quality of Balady mandarin fruits during the storage period. The storage treatments are (constant, $8^{\circ} \mathrm{C}$ and $2^{\circ} \mathrm{C}$ and $2^{\circ} \mathrm{C}$ with intermittent warming $8^{\circ} \mathrm{C}$ for 8 or 16 hours every week or biweekly). Samples of each treatments were randomly taken monthly.
\end{abstract}

Results indicated that all IW treatments decreased fruit weight loss and fruit decay percentage. The most clear reduction was associated with storage at $8^{\circ} \mathrm{C}$ or $2^{\circ} \mathrm{C}$, where the less value was related with IW treatment $\left(2^{\circ} \mathrm{C}\right.$ for 2 week followed regularly by $8^{\circ} \mathrm{C}$ for 8 hours). Also, such treatment improved fruit quality during the storage period compared to control $\left(8^{\circ} \mathrm{C}\right)$.

The fruit weight loss $\%$, fruit decay $\%$, total soluble solids $\%$, sugar contents $\%$ and TSS/acid ratio were significantly increased with advanced cold storage period. On the other hand, prolonging storage induced a gradually decrease of fruit juice percentage, acidity $\%$ and V.C. content.

It could be concluded that intermittent warming $\left(2^{\circ} \mathrm{C}\right.$ for 2 weeks plus $8^{\circ} \mathrm{C}$ for $8 \mathrm{~h}$ ) seemed to be the proper and ideal treatment to prolong cold storage of Balady mandarin fruits without great reduction in fruit quality.

Keywords: Balady mandarin, storability, intermittent warming, storage.

Received on:7/7/2014

Referees: Prof. Kamelia I. Ahmed
Accepted for publication on: 22/7/2014 Prof. Nazmy Abdel-Hamed 


\section{Introduction}

Citrus is one of the most important crops in the world. It ranks the third position between fruit crops and only preceded with grapes and apples. In Egypt, it is the most important fruit crop. Balady mandarin one of the most important citrus species that Egyptian consumers prefer because of its easily peeling than other citrus species. No doubt that process of handling and storage for local or export market is an important and of vital interest as well as fruit production and its quality, post-harvest diseases destroy $10-30 \%$ of the total yield of crop. In developing countries, the post-harvest diseases destroy more than $30 \%$ of the crop yield (Kader, 2002). Cooling citrus fruits at optimal temperature during storage may be effective to decrease blue mold, but exposure to suboptimal low chilling temperatures may cause chilling injury, which my favor the decay causing organisms. Thus longterm low temperature storage of citrus fruits is limited by their sensitively to chilling (Kader 2002, Arpaia \& Kader, 2009 and Abdurrab et al., 2012).

Storage of chilling sensitive tropical and subtropical fruits at chilling temperatures causes several physiological and biochemical changes leading to disorders. External symptoms are manifested as pitting, discolouration, water soaking, breakdown and off-flavour. Chilling injury appeared to be directly related to length of exposure to chilling and inversely related to temperature (Ladaniya and Sonkar, 1996). In order to minimize such post harvest disorders i.e. diseases and chilling injury, many investigators in the post-harvest field of fresh production are trying to im- prove fruit post-harvest shelf-life and to protect them from decay.

Intermediate warming at chilling temperature can prevent or reduce chilling injury development and maintain fruit quality for longer period (Wang, 1993).

Intermittent warming increases fruit resistance to low temperatures, enabling fruit to be stored at temperatures below the ones normally recommended and maintains fruit quality for longer periods (Kluge et al., 2003). Storage life of 'Nagpur' mandarin can be extended up to 75 days at $35^{\circ} \mathrm{C}$ with intermittent warming (Ladaniya, 2011). All intermittent warming (IW) decreased fresh weight losses, fruit decay and chilling injury than control. The best one was associated with IW treatment $\left(6 \mathrm{~d}\right.$ at $2^{\circ} \mathrm{C}+$ $6 \mathrm{~h}$ at $8^{\circ} \mathrm{C}$ ). However, IW treatments increased panel test, ascorbic acid, juice volume and juice percentage compared to (control) it constant temperature $8^{\circ} \mathrm{C}$ (Mohamed-Fatma El-Zahraa, 2011).

Therefore, this study aimed to recognize the benefit of using intermittent warming on storability of Balady mandarin fruits.

\section{Materials and Mehods:}

This study was conducted during the three successive seasons 2012, 2013 and 2014 on Balady mandarin (Citrus reticulata L.) fruits. The fruit were obtained from the Experimental Orchard of the Faculty of Agriculture, Assiut University.

The trees were forty two years old grafted on sour orange rootstocks, planted in clay soil at $5 \times 5$ meters apart. The trees were nearly similar in vigor and productivity and receiving regular horticultural practices.

Fruits were harvested in first week of January, hand packed and 
carefully brought, soon after picking to post-harvest laboratory in Agriculture Research Station in Assiut. The fruits were free from obvious defects or mechanical damage. All fruits were washed with regular tap water and soap and then rinsed with water to remove the residue of soap, then air dried. Fruits were divided into six groups then used for intermittent warming treatments. Each treatment was divided into three replicates, 10 uniform fruits were chosen at random for each replicate at all seasons and packed in perforated polyethylene bags.

The experiment comprised six treatments as followed:

1- Storage under constant temperature of $8^{\circ} \mathrm{C}$ (as a control), throughout the storage period.

2- Storage under constant temperature of $2^{\circ} \mathrm{C}$, throughout the storage period.

3- Storage under $2^{\circ} \mathrm{C}$ for 1 week followed regularly by $8^{\circ} \mathrm{C}$ for 8 hours, throughout the storage period.

4- Storage under $2^{\circ} \mathrm{C}$ for 1 week followed regularly by $8^{\circ} \mathrm{C}$ for 16 hours, throughout the storage period.

5- Storage under $2^{\circ} \mathrm{C}$ for 2 week followed regularly by $8^{\circ} \mathrm{C}$ for 8 hours, throughout the storage period.

6- Storage under $2^{\circ} \mathrm{C}$ for 2 week followed regularly by $8^{\circ} \mathrm{C}$ for 16 hours, throughout the storage period.

Fruits of each treatment were evaluated at a month interval throughout the storage period (4 months) to study the effect of different experimental treatments on physical and chemical properties as well as storability.

\section{Physical characteristics:}

1- Weight loss as a percentage from the initial weight.

2- Decay $\%$, the number of decayed fruits was calculated as percentage from the total number of each sample.
3- Peel percentage, peel percentage was calculated as a percentage from the fruit weight.

4- Juice percentage, juice content was expressed as percent of fruit weight (W/W).

\section{Chemical juice:}

1- The total soluble solids (TSS) was determined a hand refracometer.

2- Titratable acidity (TA) expressed as percentage of citric acid, against $0.1 \mathrm{~N}$ sodium hydroxide according to the A.O.A.C. (1985)

3- TSS/TA ratio

4- L-ascorbic acid (V.C.) was determined by titration against 2,6dichlorophenol indophenol blue dye, according to the A.O.A.C. (1985).

5- Sugars content $\%$, total and reducing sugars percentage were determined according to method of Lane and Eynone outlined in the A.O.A.C. (1985).

This experiment was conducted in complete randomized block design with three replicate and with factorial arrangement was followed throughout the whole work. All recorded data were tabulated and statistically analysed according to Snedecor and Cochran (1990) using new L.S.D. at the level of 0.05 in comparison between various treatment means.

Results

1- The percentage of fruit weight loss and decay fruits:

Data presented in Table (1) showed the effect of intermittent warming temperature on the percentage of weight loss and decay of Balady mandarin fruits during the cold storage in 2012, 2013 and 2014 seasons. It was obvious that results took similar trend during the three studied seasons.

Data in previously table clear that fruit weight loss, as well as, decayed fruits were markedly increased with advanced of cold storage period. These traits were slightly increased and gradually from the beginning of cold storage 
till the $4^{\text {th }}$ month. After one month of cold storage in all intermittent warming treatments the fruits were without any decay during the three studied seasons.

In response of intermittent warming (IW), it was apparent that all treatments significantly reduced the fruit weight loss percentage and undesirable fruit percentage during cold storage for four months compared with control $\left(\mathrm{T}_{1}\right.$, $\left.8^{\circ} \mathrm{C}\right)$. Using IW $\left(2\right.$ weeks at $2^{\circ} \mathrm{C}, 8 \mathrm{~h}$ at $8^{\circ} \mathrm{C}, \mathrm{T}_{5}$ ) had the best results, which gave the least percentage of fruit weight loss (4.62, $4.33 \& 4.87 \%)$ and decayed fruits (10.67, $10.67 \& 10.67 \%)$, against (6.45, $6.00 \& 6.39 \%)$ and $(25.33,24.67 \&$ $26.67 \%$ ) during the three studied seasons, respectively. The decrement percentage of weight loss percentage due to $\mathrm{T}_{5}$ compared to control $\left(\mathrm{T}_{1}\right)$ attained (28.37, 27.83 \& 23.79\%) and decayed fruits attained $(57.88,56.75 \& 60.00 \%)$ during the three studied seasons, respectively.

According to interaction effects, data in Table (1) indicated that all combinations after two month induced a significant decrease in fruit weight loss compared storage under $2^{\circ} \mathrm{C}\left(\mathrm{T}_{2}\right)$. On other hand, the decayed fruits significantly decreased after two month due to all combinations compared to control $\left(\mathrm{T}_{1}\right)$.

Moreover, using IW (T5) recorded the lowest fruit weight loss $(8.9,8.38$ \& $9.85 \%$ ) compared to the highest one $(15.35,14.63 \& 15.58 \%)$ due to $\mathrm{T}_{2}\left(2^{\circ} \mathrm{C}\right.$, constant) after fourth month of cold storage. Also, IW $\left(\mathrm{T}_{5}\right)$ recorded the least decayed fruits $(26.67,26.67 \& 26.67 \%)$, against the highest value $(53.33,53.33 \&$ $56.67 \%)$ due to $\mathrm{T}_{1}\left(8^{\circ} \mathrm{C}\right.$ constant $)$ after fourth month of cold storage.

These finding declared the importance of using intermittent warming to extend the cooling storage duration of Balady mandarin fruits.

\section{2- The percentage of peel and juice contents:}

The date introduced in Table (2) disclosed that the peel percentage slightly increased and gradually from beginning of cold storage till the $4^{\text {th }}$ month. The lowest fruit peel percentage recorded at the beginning of cold storage, but the highest ones regstrated after four month of cold storage. Contrarly, fruit juice percentage significantly decreased by extending cooling storage duration. The highest values recorded at beginning of cold storage, whereas, the least ones was recorded at the end of cold storage course.

All IW treatments tended to induce reduce the fruit peel percentage, as well as, increased the fruit juice percentage compared to control. The least significant fruit peel percentage $(29.70,30.01$ \& $29.84 \%$ ) and the highest significant fruit juice percentage $(35.06,34.27 \&$ $34.25 \%)$ were found due to use $\mathrm{T}_{5}(2 \mathrm{~W}$ at $2^{\circ} \mathrm{C}+8 \mathrm{~h}$ at $8^{\circ} \mathrm{C}$ ) during the three studied seasons, respectively. On other hand, the highest fruit peel percentage $(31.15,31.45 \& 31.06)$ and lowest fruit juice percentage $(33.62,33.10 \&$ $32.73 \%$ ) were recorded on fruits stored under $8^{\circ} \mathrm{C}$ (control) during the three studied seasons, respectively. No significant differences between IW $\left(\mathrm{T}_{5}\right)$ and other intermittent treatments. Also, no significant differences peel percentage or juice percentage of fruits that stored under any intermittent treatments, except $\left(\mathrm{T}_{5}\right)$ compared to fruit stored under control $\left(8^{\circ} \mathrm{C}\right.$ constant $)$.

The interaction between treatments and cold storage period was non significant during the three studied seasons.

\section{3- Fruit chemical properties:}

The data concerning the effect of various cold storage treatments on chemical constituents of Balady mandarin juice during 2012, 2013 and 2014 seasons are presented in Tables (3, 4 and 5). It was obvious from the data that results took similar trend during the three studied seasons.

In general view, data indicated that chemical juice quality in terms, total soluble solids, sugars contents and TSS/acid ratio significantly increased 
during storage duration up to $4^{\text {th }}$ month. On other hand, prolonging cooling storage for four month induce a gradually decrease of titratable acidity and ascorbic acid (V.C).

According of treatment effects, it is clear from the previously data that all intermittent warming failed to show any significant effects on chemical juice properties compared to store the fruits under $2^{\circ} \mathrm{C}$ or $8^{\circ} \mathrm{C}$ constant for full time of storage period. Moreover, using intermittent warming $\left(2 \mathrm{~W}\right.$ at $2^{\circ} \mathrm{C}+8^{\circ} \mathrm{C}$ for $8 \mathrm{~h}, \mathrm{~T}_{\mathbf{5}}$ ) to store the fruits induce unsignificant increase in total soluble solids, sugars contents and TSS/acid ratio compared to other storage treatments. Also, such treatment unsignificantly decreased the titratable acidity and (V.C) content compared to other treatments.

Table (1): Effect of Intermittent warming treatments on fresh weight loss \% and decay \% of Balady mandarin fruits during cold storage in 2012, 2013 and 2014 seasons.

\begin{tabular}{|c|c|c|c|c|c|c|c|c|c|c|c|c|}
\hline \multicolumn{7}{|c|}{ Fresh weight loss \% } & \multicolumn{6}{|c|}{ Decay \% } \\
\hline \multicolumn{13}{|c|}{ Storage period (month) } \\
\hline $\mathbf{T}$ & $\mathbf{P}_{\mathbf{0}}$ & $\mathbf{P}_{1}$ & $\mathbf{P}_{2}$ & $\mathbf{P}_{\mathbf{3}}$ & $\mathbf{P}_{4}$ & $\mathbf{M}$ & $\mathbf{P}_{0}$ & $\mathbf{P}_{1}$ & $\mathbf{P}_{2}$ & $\mathbf{P}_{\mathbf{3}}$ & $\mathbf{P}_{4}$ & M \\
\hline \multicolumn{13}{|c|}{ Season2012 } \\
\hline 1 & 0.00 & 3.58 & 6.15 & 10.83 & 11.68 & 6.45 & 0.00 & 10.00 & 20.00 & 43.33 & 53.33 & 25.33 \\
\hline 2 & 0.00 & 3.11 & 6.90 & 12.33 & 15.35 & 7.54 & 0.00 & 6.67 & 16.67 & 30.00 & 46.67 & 20.00 \\
\hline 3 & 0.00 & 2.86 & 5.30 & 7.13 & 9.85 & 5.03 & 0.00 & 0.00 & 16.67 & 30.00 & 33.33 & 16.00 \\
\hline 4 & 0.00 & 2.95 & 5.62 & 7.40 & 10.18 & 5.23 & 0.00 & 0.00 & 13.33 & 23.33 & 33.33 & 14.00 \\
\hline 5 & 0.00 & 2.60 & 4.80 & 6.78 & 8.90 & 4.62 & 0.00 & 0.00 & 10.00 & 16.67 & 26.67 & 10.67 \\
\hline 6 & 0.00 & 2.95 & 5.35 & 7.20 & 10.40 & 5.18 & 0.00 & 0.00 & 10.00 & 23.33 & 30.00 & 12.67 \\
\hline $\mathbf{M}$ & 0.00 & 3.01 & 5.69 & 8.61 & 11.06 & & 0.00 & 2.78 & 14.45 & 27.78 & 37.22 & \\
\hline \multicolumn{13}{|c|}{ Season2013 } \\
\hline 1 & 0.00 & 3.30 & 5.76 & 10.11 & 10.83 & 6.00 & 0.00 & 10.00 & 20.00 & 40.00 & 53.33 & 24.67 \\
\hline 2 & 0.00 & 2.84 & 6.48 & 11.76 & 14.63 & 7.14 & 0.00 & 6.67 & 20.00 & 36.67 & 46.67 & 22.00 \\
\hline 3 & 0.00 & 2.61 & 4.93 & 6.68 & 9.24 & 4.69 & 0.00 & 0.00 & 6.67 & 26.67 & 36.67 & 14.00 \\
\hline 4 & 0.00 & 2.73 & 5.25 & 6.90 & 9.52 & 4.88 & 0.00 & 0.00 & 10.00 & 23.33 & 40.00 & 14.67 \\
\hline 5 & 0.00 & 2.45 & 4.51 & 6.30 & 8.38 & 4.33 & 0.00 & 0.00 & 6.67 & 20.00 & 26.67 & 10.67 \\
\hline 6 & 0.00 & 2.76 & 5.30 & 6.78 & 9.30 & 4.83 & 0.00 & 0.00 & 6.67 & 23.33 & 36.67 & 13.33 \\
\hline $\mathbf{M}$ & 0.00 & 2.78 & 5.37 & 8.09 & 10.32 & & 0.00 & 2.78 & 11.67 & 28.33 & 40.00 & \\
\hline \multicolumn{13}{|c|}{ Season2014 } \\
\hline 1 & 0.00 & 3.28 & 6.56 & 10.31 & 11.78 & 6.39 & 0.00 & 10.00 & 23.33 & 43.33 & 56.67 & 26.67 \\
\hline 2 & 0.00 & 2.90 & 7.12 & 13.63 & 15.58 & 7.85 & 0.00 & 6.67 & 13.33 & 36.67 & 50.00 & 21.33 \\
\hline 3 & 0.00 & 2.60 & 5.56 & 8.24 & 10.15 & 5.31 & 0.00 & 0.00 & 10.00 & 26.67 & 33.33 & 14.00 \\
\hline 4 & 0.00 & 2.78 & 5.88 & 7.81 & 10.50 & 5.40 & 0.00 & 0.00 & 13.33 & 20.00 & 33.33 & 13.33 \\
\hline 5 & 0.00 & 2.40 & 4.90 & 7.18 & 9.85 & 4.87 & 0.00 & 0.00 & 6.67 & 20.00 & 26.67 & 10.67 \\
\hline 6 & 0.00 & 2.90 & 5.53 & 7.60 & 10.32 & 5.27 & 0.00 & 0.00 & 10.00 & 20.00 & 33.33 & 12.67 \\
\hline $\mathbf{M}$ & 0.00 & 2.81 & 5.92 & 9.13 & 11.36 & & 0.00 & 2.78 & 12.78 & 27.78 & 38.89 & \\
\hline \multicolumn{3}{|c|}{ New L.S.D. 0.05} & \multicolumn{2}{|c|}{ Season 2012} & 2013 & & & 2013 & \multicolumn{2}{|c|}{2014} \\
\hline \multirow{2}{*}{\multicolumn{3}{|c|}{$\begin{array}{l}\text { Treatment }(T) \\
\text { Period }(\mathrm{P})\end{array}$}} & \multicolumn{2}{|c|}{0.69} & 0.70 & \multicolumn{2}{|c|}{$\begin{array}{l}2014 \\
1.12\end{array}$} & \multicolumn{2}{|c|}{$\begin{array}{c}\text { Season } 2012 \\
280\end{array}$} & 3.99 & \multicolumn{2}{|c|}{2.00} \\
\hline & & & \multirow{2}{*}{\multicolumn{2}{|c|}{$\begin{array}{l}0.77 \\
1.53\end{array}$}} & 0.78 & \multirow{2}{*}{\multicolumn{2}{|c|}{$\begin{array}{l}1.25 \\
2.50\end{array}$}} & \multirow{2}{*}{\multicolumn{2}{|c|}{$\begin{array}{l}3.14 \\
6.27\end{array}$}} & 4.45 & \multirow{2}{*}{\multicolumn{2}{|c|}{$\begin{array}{l}2.24 \\
4.48\end{array}$}} \\
\hline \multicolumn{3}{|c|}{ Interaction } & & & 1.56 & & & & & 8.91 & & \\
\hline
\end{tabular}

(TxP)

Storage treatments (T) : $\quad 1-8 \mathrm{C}^{\circ} \quad 3-2 \mathrm{C}^{\circ}$ for $1 \mathrm{w} 8 \mathrm{C}^{\circ}$ for $8 \mathrm{~h}$

2- $2 \mathrm{C}^{\circ} \quad 4-2 \mathrm{C}^{\circ}$ for $1 \mathrm{w} 8 \mathrm{C}^{\circ}$ for $16 \mathrm{~h}$
5- $2 \mathrm{C}^{\circ}$ for $2 \mathrm{w} 8 \mathrm{C}^{\circ}$ for $8 \mathrm{~h}$ 6- $2 \mathrm{C}^{\circ}$ for $2 \mathrm{w} 8 \mathrm{C}^{\circ}$ for $16 \mathrm{~h}$ 
Table (2): Effect of Intermittent warming treatments on peel \% and juice weight \% of Balady mandarin fruits during cold storage in 2012, 2013 and 2014 seasons.

\begin{tabular}{|c|c|c|c|c|c|c|c|c|c|c|c|c|}
\hline \multicolumn{7}{|c|}{ peel \% } & \multicolumn{6}{|c|}{ Juice weight $\%$} \\
\hline \multicolumn{13}{|c|}{ Storage period (month) } \\
\hline $\mathbf{T}$ & $\mathbf{P}_{\mathbf{0}}$ & $\mathbf{P}_{1}$ & $\mathbf{P}_{2}$ & $\mathbf{P}_{3}$ & $\mathbf{P}_{4}$ & $\mathbf{M}$ & $\mathbf{P}_{\mathbf{0}}$ & $\mathbf{P}_{1}$ & $\mathbf{P}_{2}$ & $\mathbf{P}_{3}$ & $\mathbf{P}_{4}$ & $\mathbf{M}$ \\
\hline \multicolumn{13}{|c|}{ Season 2012} \\
\hline 1 & 28.84 & 30.19 & 31.80 & 32.10 & 32.83 & 31.15 & 37.70 & 35.81 & 34.10 & 30.47 & 30.00 & 33.62 \\
\hline 2 & 28.84 & 29.90 & 30.61 & 31.22 & 31.96 & 30.51 & 37.70 & 36.20 & 34.00 & 31.81 & 31.40 & 34.22 \\
\hline 3 & 28.84 & 29.43 & 30.13 & 30.68 & 31.67 & 30.15 & 37.70 & 34.50 & 33.60 & 32.90 & 32.50 & 34.24 \\
\hline 4 & 28.84 & 29.60 & 30.28 & 30.76 & 31.51 & 30.20 & 37.70 & 36.40 & 34.00 & 32.20 & 31.18 & 34.30 \\
\hline 5 & 28.84 & 29.20 & 29.61 & 29.95 & 30.88 & 29.70 & 37.70 & 36.10 & 34.90 & 33.80 & 32.80 & 35.06 \\
\hline 6 & 28.84 & 29.73 & 30.40 & 30.81 & 31.40 & 30.24 & 37.70 & 35.80 & 34.00 & 32.10 & 31.80 & 34.28 \\
\hline $\mathbf{M}$ & 28.84 & 29.68 & 30.47 & 30.92 & 31.71 & & 37.70 & 35.80 & 34.10 & 32.22 & 31.61 & \\
\hline \multicolumn{13}{|c|}{ Season 2013} \\
\hline 1 & 29.22 & 30.36 & 32.18 & 32.29 & 33.18 & 31.45 & 36.80 & 34.60 & 33.60 & 31.18 & 29.30 & 33.10 \\
\hline 2 & 29.22 & 29.92 & 30.56 & 31.30 & 32.20 & 30.64 & 36.80 & 35.50 & 32.80 & 30.33 & 29.45 & 32.98 \\
\hline 3 & 29.22 & 29.58 & 30.2 & 30.98 & 32.00 & 30.40 & 36.80 & 35.30 & 33.60 & 32.40 & 30.70 & 33.76 \\
\hline 4 & 29.22 & 29.70 & 30.38 & 30.81 & 31.85 & 30.39 & 36.80 & 34.70 & 33.30 & 30.80 & 30.18 & 33.16 \\
\hline 5 & 29.22 & 29.48 & 29.89 & 30.35 & 31.10 & 30.01 & 36.80 & 35.40 & 34.35 & 32.95 & 31.85 & 34.27 \\
\hline 6 & 29.22 & 29.80 & 30.45 & 30.87 & 31.80 & 30.43 & 36.80 & 34.90 & 33.85 & 31.28 & 30.20 & 33.41 \\
\hline $\mathbf{M}$ & 29.22 & 29.81 & 30.61 & 31.10 & 32.02 & & 36.80 & 35.07 & 33.58 & 31.49 & 30.28 & \\
\hline \multicolumn{13}{|c|}{ Season 2014} \\
\hline 1 & 29.18 & 30.22 & 31.13 & 31.60 & 33.19 & 31.06 & 35.65 & 34.30 & 33.28 & 30.60 & 29.80 & 32.73 \\
\hline 2 & 29.18 & 29.80 & 30.33 & 30.78 & 32.10 & 30.44 & 35.65 & 34.50 & 33.48 & 31.60 & 30.40 & 33.13 \\
\hline 3 & 29.18 & 29.31 & 29.70 & 30.53 & 31.93 & 30.13 & 35.65 & 35.18 & 34.10 & 32.80 & 31.70 & 33.89 \\
\hline 4 & 29.18 & 29.65 & 30.25 & 30.62 & 31.71 & 30.28 & 35.65 & 35.20 & 33.40 & 31.80 & 30.95 & 33.4 \\
\hline 5 & 29.18 & 29.43 & 29.80 & 29.88 & 30.90 & 29.84 & 35.65 & 35.30 & 34.60 & 33.10 & 32.60 & 34.25 \\
\hline 6 & 29.18 & 29.71 & 30.35 & 30.65 & 31.70 & 30.32 & 35.65 & 35.00 & 33.45 & 32.20 & 31.75 & 33.61 \\
\hline $\mathbf{M}$ & 29.18 & 29.69 & 30.26 & 30.68 & 31.92 & & 35.65 & 34.91 & 33.72 & 32.02 & 31.20 & \\
\hline \multirow{3}{*}{\multicolumn{3}{|c|}{$\begin{array}{l}\text { New L.S.D. } 0.05 \\
\text { Treatment (T) } \\
\text { Period (P) }\end{array}$}} & \multicolumn{2}{|c|}{ Season 2012} & \multicolumn{2}{|c|}{2013} & & \multicolumn{2}{|c|}{ Season 2012} & \multicolumn{2}{|c|}{2013} & 2014 \\
\hline & & & \multicolumn{2}{|c|}{1.24} & \multirow{2}{*}{\multicolumn{2}{|c|}{$\begin{array}{l}1.22 \\
1.36\end{array}$}} & & \multirow{2}{*}{\multicolumn{2}{|c|}{1.13}} & \multirow{2}{*}{\multicolumn{2}{|c|}{$\begin{array}{l}1.08 \\
1.20\end{array}$}} & 1.36 \\
\hline & & & & & & & & & & & & 1.51 \\
\hline \multicolumn{3}{|c|}{ nteraction (TxP) } & \multicolumn{2}{|c|}{ N.S } & \multicolumn{2}{|c|}{ N.S } & J.S & \multicolumn{2}{|c|}{ N.S } & \multicolumn{2}{|c|}{ N.S } & N.S \\
\hline
\end{tabular}

Storage treatments (T) : $\quad 1-8 \mathrm{C}^{\circ} \quad 3-2 \mathrm{C}^{\circ}$ for $1 \mathrm{w} 8 \mathrm{C}^{\circ}$ for $8 \mathrm{~h} \quad 5-2 \mathrm{C}^{\circ}$ for $2 \mathrm{w} 8 \mathrm{C}^{\circ}$ for $8 \mathrm{~h}$ 2- $2 \mathrm{C}^{\circ} \quad 4-2 \mathrm{C}^{\circ}$ for $1 \mathrm{w} 8 \mathrm{C}^{\circ}$ for $16 \mathrm{~h} \quad 6-2 \mathrm{C}^{\circ}$ for $2 \mathrm{w} 8 \mathrm{C}^{\circ}$ for $16 \mathrm{~h}$ 
Table (3): Effect of Intermittent warming treatments on total soluble solids percentage (TSS) \% and titratable acidity \% (TA) (as grams of citric acid/100 ml juice) of Balady mandarin fruits during cold storage in 2012, 2013 and 2014 seasons.

\begin{tabular}{|c|c|c|c|c|c|c|c|c|c|c|c|c|}
\hline \multicolumn{7}{|c|}{ TSS \% } & \multicolumn{6}{|c|}{ TA \% } \\
\hline \multicolumn{13}{|c|}{ Storage period (month) } \\
\hline $\mathbf{T}$ & $\mathbf{P}_{\mathbf{0}}$ & $\mathbf{P}_{1}$ & $\mathbf{P}_{2}$ & $\mathbf{P}_{3}$ & $\mathbf{P}_{4}$ & $\mathbf{M}$ & $\mathbf{P}_{0}$ & $\mathbf{P}_{1}$ & $\mathbf{P}_{2}$ & $\mathbf{P}_{3}$ & $\mathbf{P}_{4}$ & $\mathbf{M}$ \\
\hline \multicolumn{13}{|c|}{ Season 2012} \\
\hline 1 & 10.20 & 10.53 & 11.30 & 11.60 & 12.40 & 11.21 & 1.25 & 1.21 & 0.96 & 0.83 & 0.78 & 1.01 \\
\hline 2 & 10.20 & 10.40 & 11.20 & 11.70 & 12.10 & 11.12 & 1.25 & 1.24 & 0.96 & 0.84 & 0.80 & 1.02 \\
\hline 3 & 10.20 & 10.50 & 11.30 & 11.50 & 11.93 & 11.09 & 1.25 & 1.12 & 0.88 & 0.81 & 0.75 & 0.96 \\
\hline 4 & 10.20 & 10.60 & 11.80 & 11.90 & 12.30 & 11.36 & 1.25 & 1.08 & 0.91 & 0.82 & 0.77 & 0.97 \\
\hline 5 & 10.20 & 10.62 & 11.88 & 12.00 & 12.50 & 11.44 & 1.25 & 1.05 & 0.98 & 0.85 & 0.79 & 0.98 \\
\hline 6 & 10.20 & 10.43 & 11.50 & 11.88 & 12.00 & 11.20 & 1.25 & 1.12 & 0.96 & 0.84 & 0.79 & 0.99 \\
\hline $\mathbf{M}$ & 10.20 & 10.51 & 11.50 & 11.76 & 12.21 & & 1.25 & 1.14 & 0.94 & 0.83 & 0.78 & \\
\hline \multicolumn{13}{|c|}{ Season 2013} \\
\hline 1 & 11.00 & 11.50 & 12.15 & 12.40 & 13.10 & 12.03 & 1.32 & 1.15 & 1.03 & 0.93 & 0.84 & 1.05 \\
\hline 2 & 11.00 & 11.53 & 12.00 & 12.80 & 13.00 & 12.07 & 1.32 & 1.18 & 1.10 & 0.96 & 0.89 & 1.09 \\
\hline 3 & 11.00 & 11.27 & 12.00 & 12.47 & 13.00 & 11.95 & 1.32 & 1.05 & 1.02 & 0.88 & 0.85 & 1.02 \\
\hline 4 & 11.00 & 10.85 & 12.10 & 12.30 & 12.80 & 11.81 & 1.32 & 1.03 & 1.00 & 0.88 & 0.85 & 1.02 \\
\hline 5 & 11.00 & 11.60 & 12.31 & 12.83 & 13.25 & 12.20 & 1.32 & 1.10 & 1.03 & 0.90 & 0.83 & 1.04 \\
\hline 6 & 11.00 & 11.30 & 11.95 & 12.40 & 12.93 & 11.92 & 1.32 & 1.05 & 0.91 & 0.83 & 0.81 & 0.98 \\
\hline $\mathbf{M}$ & 11.00 & 11.34 & 12.09 & 12.53 & 13.01 & & 1.32 & 1.09 & 1.02 & 0.90 & 0.85 & \\
\hline \multicolumn{13}{|c|}{ Season 2014} \\
\hline 1 & 11.33 & 11.60 & 11.85 & 12.20 & 12.68 & 11.93 & 1.28 & 1.20 & 1.02 & 0.90 & 0.83 & 1.05 \\
\hline 2 & 11.33 & 11.70 & 11.70 & 12.60 & 12.83 & 12.03 & 1.28 & 1.18 & 1.03 & 0.90 & 0.85 & 1.05 \\
\hline 3 & 11.33 & 11.38 & 12.00 & 12.20 & 12.80 & 11.94 & 1.28 & 1.09 & 1.00 & 0.88 & 0.81 & 1.01 \\
\hline 4 & 11.33 & 11.50 & 11.80 & 12.00 & 12.30 & 11.79 & 1.28 & 1.03 & 0.96 & 0.86 & 0.78 & 0.98 \\
\hline 5 & 11.33 & 11.83 & 11.85 & 12.80 & 12.98 & 12.16 & 1.28 & 1.00 & 0.93 & 0.82 & 0.76 & 0.96 \\
\hline 6 & 11.33 & 11.55 & 11.83 & 12.13 & 12.38 & 11.84 & 1.28 & 1.00 & 0.98 & 0.88 & 0.82 & 0.99 \\
\hline $\mathbf{M}$ & 11.33 & 11.59 & 11.84 & 12.32 & 12.66 & & 1.28 & 1.08 & 0.99 & 0.87 & 0.81 & \\
\hline \multirow{3}{*}{\multicolumn{3}{|c|}{$\begin{array}{l}\text { New L.S.D. } 0.05 \\
\text { Treatment (T) } \\
\text { Period (P) }\end{array}$}} & \multicolumn{2}{|c|}{ Season 2012} & \multicolumn{2}{|c|}{2013} & \multicolumn{3}{|c|}{ Season 2012} & 2013 & \multicolumn{2}{|c|}{2014} \\
\hline & & & \multirow{2}{*}{\multicolumn{2}{|c|}{$\begin{array}{l}\text { N.S } \\
1.33\end{array}$}} & N.S & & N.S & N.S & & N.S & \multicolumn{2}{|c|}{ N.S } \\
\hline & & & & & 0.82 & & 0.57 & 0.09 & & 0.09 & \multicolumn{2}{|c|}{0.13} \\
\hline \multicolumn{3}{|c|}{$\begin{array}{l}\text { Interaction }(\mathbf{T x P}) \\
\text { Storage treatments }\end{array}$} & \multicolumn{2}{|c|}{ N.S } & N.S & $\mathrm{N}$ & $S$ & N.S & & N.S & \multirow{2}{*}{\multicolumn{2}{|c|}{$\begin{array}{c}\text { N.S } \\
\text { w } 8 \mathrm{C}^{\circ} \text { for } 8 \mathrm{~h} \\
\mathrm{w} 8 \mathrm{C}^{\circ} \text { for } 16 \mathrm{~h}\end{array}$}} \\
\hline \multicolumn{4}{|c|}{ Storage treatments $(T):$} & $\begin{array}{l}1-8 C^{\circ} \\
2-2 C^{\circ}\end{array}$ & & $\begin{array}{l}-2 \mathrm{C}^{\circ} \mathrm{fc} \\
-2 \mathrm{C}^{\circ} \mathrm{fc}\end{array}$ & $\begin{array}{l}\text { or } 1 \mathrm{w} 8 \\
\text { or } 1 \mathrm{w} 8\end{array}$ & $\begin{array}{l}\mathrm{C}^{\circ} \text { for } 81 \\
\mathrm{C}^{\circ} \text { for } 1(\end{array}$ & & $\begin{array}{l}-2 \mathrm{C}^{\circ} \mathrm{fc} \\
-2 \mathrm{C}^{\circ} \mathrm{fc}\end{array}$ & & \\
\hline
\end{tabular}


Table (4): Effect of Intermittent warming treatments on TSS/TA ratio and vitamin $C$ content ( $\mathrm{mg}$ Ascorbic acid/100 $\mathrm{ml}$ juice) of Balady mandarin fruits during cold storage in 2012, 2013 and 2014 seasons.

\begin{tabular}{|c|c|c|c|c|c|c|c|c|c|c|c|c|}
\hline \multicolumn{7}{|c|}{ TSS/TA ratio } & \multicolumn{6}{|c|}{ vitamin $C$ content } \\
\hline \multicolumn{13}{|c|}{ Storage period (month) } \\
\hline$T$ & $\mathbf{P}_{\mathbf{0}}$ & $\mathbf{P}_{1}$ & $\mathbf{P}_{2}$ & $\mathbf{P}_{3}$ & $\mathbf{P}_{4}$ & $\mathbf{M}$ & $\mathbf{P}_{0}$ & $\mathbf{P}_{1}$ & $\mathbf{P}_{2}$ & $\mathbf{P}_{3}$ & $\mathbf{P}_{4}$ & $\mathbf{M}$ \\
\hline \multicolumn{13}{|c|}{ Season 2012} \\
\hline 1 & 8.16 & 8.70 & 11.77 & 13.98 & 15.90 & 11.70 & 43.3 & 36.38 & 34.60 & 33.34 & 32.31 & 35.99 \\
\hline 2 & 8.16 & 8.39 & 11.67 & 13.93 & 15.13 & 11.45 & 43.3 & 37.10 & 35.82 & 33.65 & 32.80 & 36.53 \\
\hline 3 & 8.16 & 9.38 & 12.84 & 14.20 & 15.91 & 12.10 & 43.3 & 38.00 & 36.50 & 34.10 & 33.12 & 37.00 \\
\hline 4 & 8.16 & 9.82 & 12.97 & 14.51 & 15.97 & 12.29 & 43.3 & 37.24 & 36.10 & 33.80 & 32.85 & 36.66 \\
\hline 5 & 8.16 & 10.11 & 12.12 & 14.12 & 15.82 & 12.07 & 43.3 & 38.80 & 37.36 & 34.90 & 33.93 & 37.66 \\
\hline 6 & 8.16 & 9.31 & 11.98 & 14.14 & 15.19 & 11.76 & 43.3 & 37.15 & 36.42 & 34.18 & 33.10 & 36.83 \\
\hline M & 8.16 & 9.28 & 12.22 & 14.15 & 15.65 & & 43.3 & 37.45 & 36.13 & 33.99 & 33.02 & \\
\hline \multicolumn{13}{|c|}{ Season 2013} \\
\hline 1 & 8.33 & 10.00 & 11.80 & 13.33 & 15.60 & 11.81 & 40.00 & 34.60 & 32.73 & 31.58 & 30.37 & 33.86 \\
\hline 2 & 8.33 & 9.77 & 10.91 & 13.33 & 14.61 & 11.39 & 40.00 & 35.25 & 34.00 & 31.90 & 31.18 & 34.47 \\
\hline 3 & 8.33 & 10.73 & 11.76 & 14.17 & 15.29 & 12.06 & 40.00 & 35.86 & 34.51 & 32.35 & 31.40 & 34.82 \\
\hline 4 & 8.33 & 10.53 & 12.10 & 13.98 & 15.06 & 12.00 & 40.00 & 35.31 & 34.20 & 32.11 & 31.13 & 34.55 \\
\hline 5 & 8.33 & 10.54 & 11.95 & 14.26 & 15.96 & 12.21 & 40.00 & 36.75 & 35.30 & 32.90 & 31.83 & 35.36 \\
\hline 6 & 8.33 & 10.76 & 13.13 & 14.94 & 15.96 & 12.63 & 40.00 & 35.50 & 34.60 & 32.36 & 31.41 & 34.77 \\
\hline $\mathbf{M}$ & 8.33 & 10.39 & 11.94 & 14.00 & 15.41 & & 40.00 & 35.55 & 34.22 & 32.2 & 31.22 & \\
\hline \multicolumn{13}{|c|}{ Season 2014} \\
\hline 1 & 8.85 & 9.67 & 11.62 & 13.56 & 15.28 & 11.79 & 41.70 & 34.85 & 33.38 & 32.41 & 31.10 & 34.69 \\
\hline 2 & 8.85 & 9.92 & 11.36 & 14.00 & 15.09 & 11.84 & 41.70 & 35.51 & 34.41 & 32.70 & 31.80 & 35.22 \\
\hline 3 & 8.85 & 10.44 & 12.00 & 13.86 & 15.80 & 12.19 & 41.70 & 36.11 & 35.20 & 33.22 & 32.00 & 35.65 \\
\hline 4 & 8.85 & 11.17 & 12.29 & 13.95 & 15.77 & 12.41 & 41.70 & 35.38 & 34.78 & 32.93 & 31.81 & 35.32 \\
\hline 5 & 8.85 & 11.83 & 12.74 & 15.61 & 17.08 & 13.22 & 41.70 & 37.80 & 36.10 & 33.75 & 32.40 & 36.35 \\
\hline 6 & 8.85 & 11.55 & 12.07 & 13.78 & 15.10 & 12.27 & 41.70 & 35.83 & 35.00 & 33.14 & 32.00 & 35.53 \\
\hline $\mathbf{M}$ & 8.85 & 10.76 & 12.01 & 14.13 & 15.69 & & 41.70 & 35.91 & 34.81 & 33.03 & 31.85 & \\
\hline \multirow{3}{*}{\multicolumn{3}{|c|}{$\begin{array}{c}\text { New L.S.D. } 0.05 \\
\text { Treatment (T) } \\
\text { Period (P) }\end{array}$}} & \multicolumn{2}{|c|}{ Season 2012} & & \multicolumn{2}{|c|}{2014} & \multicolumn{2}{|c|}{ Season 2012} & 2013 & \multicolumn{2}{|c|}{2014} \\
\hline & & & \multicolumn{2}{|c|}{ N.S } & & \multicolumn{2}{|c|}{ N.S } & \multicolumn{2}{|c|}{ N.S } & N.S & \multicolumn{2}{|r|}{ N.S } \\
\hline & & & & 1.40 & & \multirow{2}{*}{\multicolumn{2}{|c|}{$\begin{array}{l}2.02 \\
\text { N.S }\end{array}$}} & \multicolumn{2}{|l|}{4.00} & 3.98 & \multicolumn{2}{|r|}{2.92} \\
\hline \multicolumn{3}{|c|}{ Interaction (TxP) } & & N.S & N.s & & & N.S & & N.S & & N.S \\
\hline
\end{tabular}

Storage treatments (T) : $\quad 1-8 \mathrm{C}^{\circ} \quad 3-2 \mathrm{C}^{\circ}$ for $1 \mathrm{w} 8 \mathrm{C}^{\circ}$ for $8 \mathrm{~h}$ 5- $2 \mathrm{C}^{\circ}$ for $2 \mathrm{w} 8 \mathrm{C}^{\circ}$ for $8 \mathrm{~h}$ 2- $2 \mathrm{C}^{\circ} \quad 4-2 \mathrm{C}^{\circ}$ for $1 \mathrm{w} 8 \mathrm{C}^{\circ}$ for $16 \mathrm{~h}$ 6- $2 \mathrm{C}^{\circ}$ for $2 \mathrm{w} 8 \mathrm{C}^{\circ}$ for $16 \mathrm{~h}$ 
Table (5): Effect of intermittent warming treatments on total sugar and reducing sugar of Balady mandarin fruits during cold storage in 2012, 2013 and 2014 seasons.

\begin{tabular}{|c|c|c|c|c|c|c|c|c|c|c|c|c|}
\hline \multicolumn{7}{|c|}{ total sugar } & \multicolumn{6}{|c|}{ Reducing sugar } \\
\hline \multicolumn{13}{|c|}{ Storage period (month) } \\
\hline $\mathbf{T}$ & $\mathbf{P}_{\mathbf{0}}$ & $\mathbf{P}_{1}$ & $\mathbf{P}_{2}$ & $\mathbf{P}_{3}$ & $\mathbf{P}_{4}$ & $\mathbf{M}$ & $\mathbf{P}_{\mathbf{0}}$ & $\mathbf{P}_{1}$ & $\mathbf{P}_{2}$ & $\mathbf{P}_{3}$ & $\mathbf{P}_{4}$ & $\mathbf{M}$ \\
\hline \multicolumn{13}{|c|}{ Season 2012} \\
\hline 1 & 7.25 & 7.63 & 8.21 & 8.48 & 8.85 & 8.08 & 2.96 & 3.15 & 3.40 & 3.55 & 3.68 & 3.35 \\
\hline 2 & 7.25 & 7.52 & 8.13 & 8.51 & 8.60 & 8.00 & 2.96 & 3.11 & 3.37 & 3.58 & 3.95 & 3.39 \\
\hline 3 & 7.25 & 7.60 & 8.23 & 8.43 & 8.72 & 8.05 & 2.96 & 3.18 & 3.43 & 3.46 & 3.98 & 3.40 \\
\hline 4 & 7.25 & 7.68 & 8.46 & 8.53 & 8.80 & 8.14 & 2.96 & 3.21 & 3.51 & 3.50 & 3.75 & 3.39 \\
\hline 5 & 7.25 & 7.62 & 8.51 & 8.53 & 9.13 & 8.21 & 2.96 & 3.15 & 3.56 & 3.58 & 4.02 & 3.45 \\
\hline 6 & 7.25 & 7.50 & 8.42 & 8.50 & 8.86 & 8.11 & 2.96 & 3.18 & 3.50 & 3.53 & 3.82 & 3.40 \\
\hline $\mathbf{M}$ & 7.25 & 7.59 & 8.33 & 8.50 & 8.83 & & 2.96 & 3.16 & 3.46 & 3.53 & 3.87 & \\
\hline \multicolumn{13}{|c|}{ Season 2013} \\
\hline 1 & 7.92 & 8.32 & 8.84 & 9.03 & 9.53 & 8.73 & 3.35 & 3.53 & 3.75 & 3.87 & 4.10 & 3.72 \\
\hline 2 & 7.92 & 8.36 & 8.76 & 9.28 & 9.41 & 8.75 & 3.35 & 3.59 & 3.71 & 3.96 & 4.08 & 3.74 \\
\hline 3 & 7.92 & 8.30 & 8.80 & 9.25 & 9.48 & 8.75 & 3.35 & 3.48 & 3.75 & 3.93 & 4.08 & 3.72 \\
\hline 4 & 7.92 & 8.28 & 8.85 & 9.10 & 9.36 & 8.70 & 3.35 & 3.45 & 3.80 & 3.88 & 4.03 & 3.70 \\
\hline 5 & 7.92 & 8.36 & 8.86 & 9.30 & 9.53 & 8.79 & 3.35 & 3.61 & 3.78 & 3.98 & 4.08 & 3.76 \\
\hline 6 & 7.92 & 8.31 & 8.75 & 9.18 & 9.41 & 8.71 & 3.35 & 3.51 & 3.73 & 3.86 & 4.06 & 3.70 \\
\hline $\mathbf{M}$ & 7.92 & 8.32 & 8.81 & 9.19 & 9.45 & & 3.35 & 3.53 & 3.75 & 3.91 & 4.07 & \\
\hline \multicolumn{13}{|c|}{ Season 2014} \\
\hline 1 & 8.10 & 8.32 & 8.53 & 8.80 & 9.20 & 8.59 & 3.18 & 3.29 & 3.38 & 3.53 & 3.70 & 3.42 \\
\hline 2 & 8.10 & 8.41 & 8.48 & 9.02 & 9.28 & 8.66 & 3.18 & 3.34 & 3.36 & 3.60 & 3.73 & 3.44 \\
\hline 3 & 8.10 & 8.22 & 8.58 & 8.84 & 9.22 & 8.59 & 3.18 & 3.25 & 3.41 & 3.55 & 3.70 & 3.42 \\
\hline 4 & 8.10 & 8.26 & 8.55 & 8.86 & 8.98 & 8.55 & 3.18 & 3.28 & 3.40 & 3.53 & 3.61 & 3.40 \\
\hline 5 & 8.10 & 8.43 & 8.63 & 9.05 & 9.31 & 8.70 & 3.18 & 3.33 & 3.43 & 3.61 & 3.74 & 3.46 \\
\hline 6 & 8.10 & 8.31 & 8.53 & 8.90 & 9.03 & 8.57 & 3.18 & 3.30 & 3.36 & 3.55 & 3.60 & 3.40 \\
\hline $\mathbf{M}$ & 8.10 & 8.33 & 8.55 & 8.92 & 9.17 & & 3.18 & 3.30 & 3.39 & 3.56 & 3.68 & \\
\hline \multirow{3}{*}{\multicolumn{3}{|c|}{$\begin{array}{l}\text { New L.S.D. } 0.05 \\
\text { Treatment (T) } \\
\text { Period (P) }\end{array}$}} & \multicolumn{2}{|c|}{ Season 2012} & \multicolumn{2}{|c|}{2013} & & \multicolumn{2}{|c|}{ Season 2012} & \multicolumn{2}{|l|}{2013} & 2014 \\
\hline & & & \multicolumn{2}{|c|}{ N.S } & \multirow{2}{*}{\multicolumn{2}{|c|}{$\begin{array}{l}\text { N.S } \\
0.94\end{array}$}} & & \multicolumn{2}{|c|}{ N.S } & \multicolumn{2}{|l|}{ N.S } & N.S \\
\hline & & & \multirow{2}{*}{\multicolumn{2}{|c|}{ N.S }} & & & & \multicolumn{2}{|c|}{0.38} & \multicolumn{2}{|l|}{0.41} & 0.36 \\
\hline \multicolumn{3}{|c|}{ nteraction (TxP) } & & & \multicolumn{2}{|c|}{ N.S } & $S$ & N.S & & N.S & & N.S \\
\hline
\end{tabular}

Storage treatments (T) : $\quad 1-8 \mathrm{C}^{\circ} \quad 3-2 \mathrm{C}^{\circ}$ for $1 \mathrm{w} 8 \mathrm{C}^{\circ}$ for $8 \mathrm{~h} \quad 5-2 \mathrm{C}^{\circ}$ for $2 \mathrm{w} 8 \mathrm{C}^{\circ}$ for $8 \mathrm{~h}$ 2- $2 \mathrm{C}^{\circ} \quad 4-2 \mathrm{C}^{\circ}$ for $1 \mathrm{w} 8 \mathrm{C}^{\circ}$ for $16 \mathrm{~h} \quad 6-2 \mathrm{C}^{\circ}$ for $2 \mathrm{w} 8 \mathrm{C}^{\circ}$ for $16 \mathrm{~h}$ 
The interaction between treatments and cold storage period was insignificant for all studied chemical properties in three studied seasons. Moreover, the highest values of total soluble solids, sugar contents, TSS/acid ratio and V.C. contents were recorded on fruits stored under $\left(\mathrm{T}_{5}\right)$ at end of storage period compared with other interaction treatments.

According to previous results, it could be concluded that intermittent warming (IW) especially storage un$\operatorname{der} 2^{\circ} \mathrm{C}$ for 2 weeks followed regularly by $8^{\circ} \mathrm{C}$ for 8 hours $\left(\mathrm{T}_{5}\right)$ seemed to be the proper and ideal treatment to prolong cold storage of mandarin fruits without great reduction in fruit quality.

\section{4- Storability of Balady mandarin fruits:}

Data presented in Figure (1) show the effect of certain intermittent warming on storability of Balady mandarin fruits as average of three studied seasons. According to weight loss increased in a gradual manner from the beginning or storage till the end of it. It was generally higher at low chilling temperature $\left(2^{\circ}\right)$. The percentage of weight loss of fruits stored at $2^{\circ} \mathrm{C}$ was $15.19 \%$ after four months of storage, which decreased to 11.43 and $9.04 \%$ for fruits stored at $8^{\circ} \mathrm{C}$ (as control) and at $2^{\circ} \mathrm{C}$ with intermittent warming with regularly $8^{\circ} \mathrm{C}$ for 8 hours every two weeks, respectively. The corresponding weight loss after three months of storage attained $(12.57,10.97$ and $6.75 \%)$, respectively. Increased weight loss with increasing storage is common in fruits. The increased rate of weight loss in fruits exposed to low temperature may be due to their higher moisture content due low temperature storage and hence having the potential to loss more water but also due to chilling related damage to the rind of such fruits (Lindhout et al., 2004).

Also, data in Figure (1) indicated that decayed fruits percentage consistently enhanced with increased the storage period. Decay percentage was lower for fruits stored under intermittent warming compared to fruits stored either at $2^{\circ} \mathrm{C}$ or $8^{\circ} \mathrm{C}$. The decay percentage attained $(54.45$, $47.78 \& 26.67 \%$ av. the three studied seasons) due to store fruits at $8^{\circ} \mathrm{C}$, $2{ }^{\circ} \mathrm{C}$ and intermittent warming, respectively. The corresponding decay percentage after three months of storage attained $(42.22,34.45$ and $18.89 \%$ ), respectively. Decreasing the decayed fruits under intermittent warming due to its role in prevent or reduce chilling injury development and maintain fruit quality for longer period (Wang, 1993). So, according to weight loss and decay percentage which effect on peel shrink and appearance of fruits. It could be concluded that the Balady mandarin fruits could be stored for three months under $2^{\circ} \mathrm{C}$ or $8^{\circ} \mathrm{C}$ and more four months under intermittent warming. Extending storage period more than three months for storage at $2^{\circ} \mathrm{C}$ and $8^{\circ} \mathrm{C}$ produced fruits unsuitable for consumer acceptance either fresh fruits or as juice stage. 

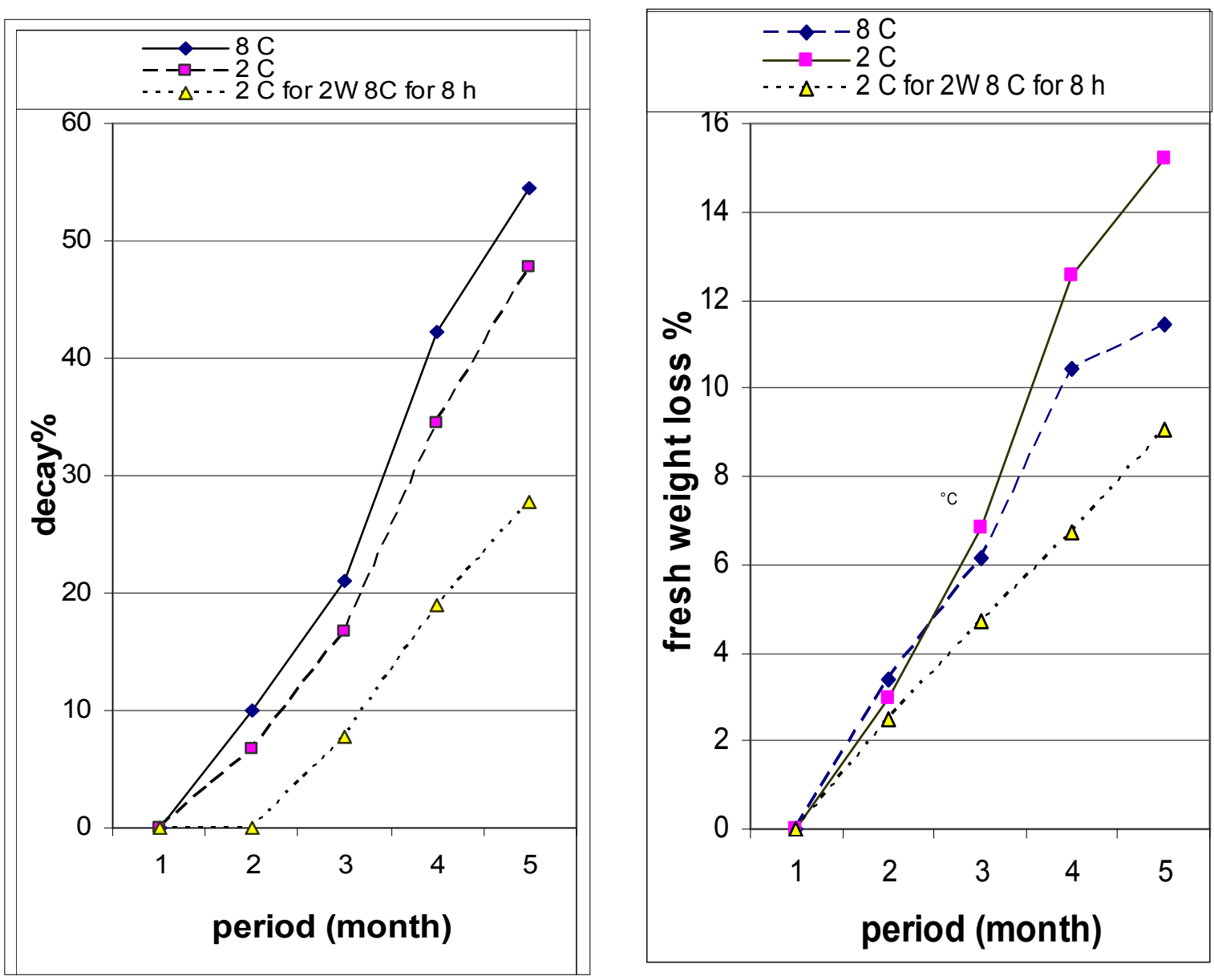

Fig (1): Effect of storage period and treatments on fresh weight loss and decay \% changes.

\section{Discussion}

Handling and storage is an important and of vital interest to fruit production and its quality. Cooling citrus fruits at optimal temperature during storage is effected to pathological breakdown, but exposure to low chilling temperatures cause chilling injury, which may favor the decay causing organisms. Thus long-term low temperature storage of citrus fruits is limited since their sensitively to chilling (Arpaia \& Kader, 2009 and Abdurrab, 2012).

Fresh weight loss and decayed fruit percentage were increased by extending cooling storage duration. Increased weight loss with increasing storage is common in fruits, but the increased rate of weight loss in fruits exposed to low temperature may be due to their higher moisture content due low temperature storage and hence having the potential to lose more water but also due to chilling related damage to the rind of such fruits (Lindhout et al., 2004).

Intermittent warming (IW) at chilling temperature can prevent or reduce chilling injury development and maintain fruit quality for longer period (Wang, 1993 and Kluge et al., 2003).

The reduction of weight loss and decay due to store under intermittent warming may be to its role in prevent or reduce chilling injury. These results are in accordance with those obtained by Martinez et al. (1994); Schirra and Mulas (1995); 
Ladaniya et al. (2005); MohamedFatma El-Zahraa (2011) and Abdurrab (2012). They revealed that use intermittent warming with low temperature decrease the weight loss and decayed fruit and maintains fruit quality for longer periods.

Juice content, titratable acidity and ascorbic acid (V.C.) content were significantly declined with extended storage duration. The rate of decreases in these traits were low due to use intermittent warming (IW). Ascorbic acid content is an important constituent of different fruits and the retention of it has a major importance during post-harvest handling of citrus fruits. Ascorbic acid losses to be associated with chilling injury symptom development (Ladaniya, 2011).

Chilling temperature causes stress and accumulation of toxic metabolites resulting in injury but IW results in depletion of such metabolites before the occurrence of degenerative change (Wang, 1993).

On other hand, totals soluble solids (TSS, TSS/acid ratio) and sugar contents were increased gradually as prolong the storage period. TSS and sugar contents were high in fruits stored under IW treatments. This increase could be due to solubilization of compounds other than carbohdyates or to some extent concentration effects. Whereas, the increment of reducing sugar could be due to the increase of activity of invertase enzyme. In addition, the improve in the TSS/acid ratio since the slight increase in TSS and higher decrease in acidity with the progress of storage period, or stored at intermittent warming (Ladaniya, 2011).

The above mentioned findings are in accordance with those obtained by Artes et al. (1993), Schirra and
Mulas (1995), El-Hefnawi (2002), Ladaniya et al. (2005), Mahajan et al. (2006), Ladaniya (2011), MohamedFatma El-Zahraa (2011) and Abdurrab (2012). They concluded that all fruit quality unsignificantly improve when stored under intermittent warming. On the other hand, TSS and sugar contents significantly increased with advanced cold storage period, whereas, total acidity and V.C. content were gradually and significantly decreased.

\section{Conclusion:}

According to the previous results, it could be concluded that storage of the Balady mandarin fruits under $2^{\circ} \mathrm{C}$ with intermittent warming with regularly $8^{\circ} \mathrm{C}$ for 8 hours every two weeks would keep the fruits and maintain their quality for longest period.

\section{References:}

Abdurrab, M. Sajed; N. Khan; K. Nawab; M. Arif and MK. Khattak (2012): Influence of storage temperature on fungal prevalence and quality of citrus fruit (cv. Blood Red). Pak. J. Bot., 44 (2): 831-836.

AOAC (1985): Official Method Analysis. Association of Official Analytical Chemists, Washington, DC., USA.

Arpaia, M.L. and A.A. Kader (2009): Orange: recommendation for maintaining quality UCD. Postharvest Fact Sheet UC Davis, USA.

Artes, F.; A.J. Escriche and J.G. Marin (1993): Treating "Primofiori" lemons in cold storage with intermittent warming and carbon dioxide. HortScience, 28 (8): 819-821.

El-Hefnawi， S.M. (2002): Intermittent warming effect on post- 
harvest cold storage of Baladi lime fruits. Hort. Dept., Fac. Agric., Zagazig Univ., Egypt, 29 (3): 727-744.

Kader, A.A. (2002): Modified atmospheres during transport and storage. In: post-harvest technology of Horticultural Crops (Ed.) A.A. Kadder, Univ. Calif. Div. Agr. Nat. Res. Publ. 3311. Oakland, CA pp. 135-144.

Kluge, R.A.; L.L. Maria-Jomoria; A.P. Jacomino; D.V. MariaCarolina and M. Padula (2003): Intermittent warming in "Tahiti" lime treated with an ethylene inhibitor. Post-harvest Biol. Technol., 29: 195-203.

Ladaniya, M.S. (2011): Physicochemical, respiratory and fungicide residue changes in wax coated mandarin fruit stored at chilling temperature with intermittent warming. J. Food Sci. Technol., 48 (2): 150-158.

Ladaniya, M.S. and R.K. Sonkar (1996): Influence of temperature and fruit maturity on "Nagpur" mandarin (Citrus reticulata Blanco) in storage. Indian J. Agric. Sci. 66: 109-113.

Ladaniya, M.S.; Singh Shyam and B. Mahalle (2005): Suboptimum low temperature storage of "Nagpur" mandarin as influenced by wax coating and intermittent warming. Indian J. of Hort., 62 (1): 1-7.
Lindhout, K.; M.T. Treeby and R.W. Parish (2004): Chilling related injuries in Navel orange SHS Acta Hort. 687.

Mahajan, B.V.C.; A.S. Dhatt; S. Kumar and L. Manohar (2006): Effect of pre-storage treatments and packaging on storage behaviour and quality of Kinnow mandarin. J. Food Sci. Technol. 43: 589-593.

Mohamed-Fatma El-Zahraa, E. (2011): Impact of intermittent warming on storage and shelf life on mandarin fruits. M.Sc. Fac. of Agric., Zagazig Univ., Egypt, pp. 61.

Martinez, J.M.; M.A. Rio; M. Mateos and C. Saucedo (1994): Influence of storage temperature and coating on the keeping quality of "Fortune" mandarins. International Society of Citriculture, Catania, Italy, 1102-1103.

Schirra, M. and M. Mulas (1995): "Fortune" mandarin quality following pre-storage water dips and intermittent warming during cold storage. HortScience, 30 (3): 560-561.

Snedecor, G.W. and W.G. Cochran (1990): Statistical Methods $7^{\text {th }}$ Edn, Iowa State University Press, Ames, IA, USA

Wang, C.Y. (1993): Approaches to reduce chilling injury of fruits and vegetables. Hortic. Rev. 15: 63-95. 
تأثير معاملات التدفئة المتقطعة علي القدرة التخزينية لثمار اليوسفي البلاي

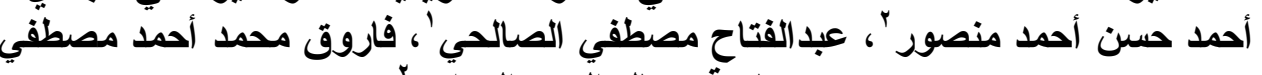

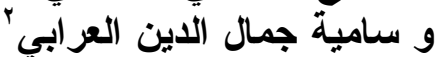

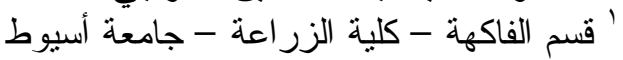

ץ مركز البحوث الزر اعية - معهد بحوث البساتينة البعة - الجيزه

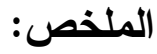

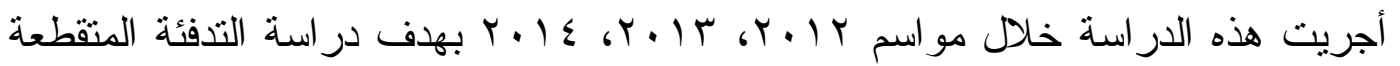

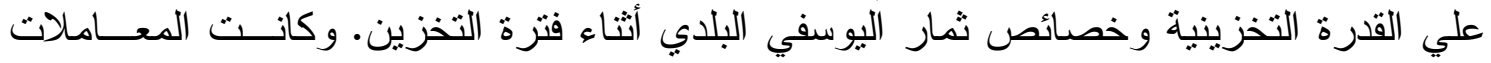
كالتالي: علي - - التخزين علي درجة حر ارة ^^م (معاملة المقارنة) طو ال فترة التخزين.

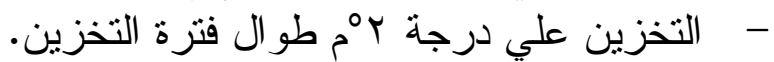

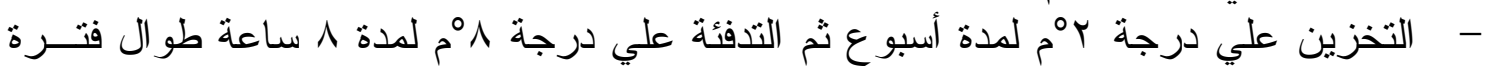

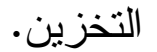

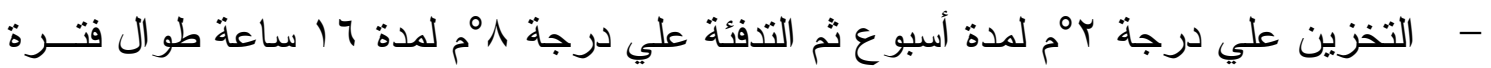
التخزين. - - التخزين علي درجة بمّم لمدة أسبوعين ثم التدفئة علي درجة ^^م لمدة ^ ساعة طو ال فترة التخزين.

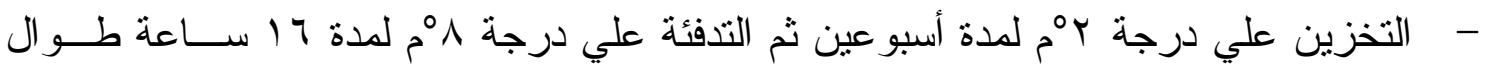
فترة التخزين. وقد أوضحت النتائج:

ا - حدوث نقص تدريجي للرطوبة و الثمار التالفة ونسبة العصبر و الحموضة ومحتهـوي الثــــار

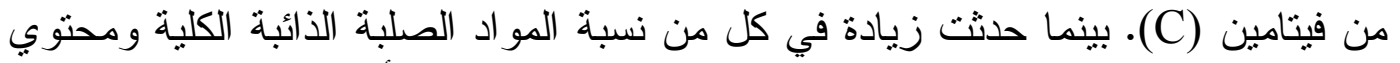

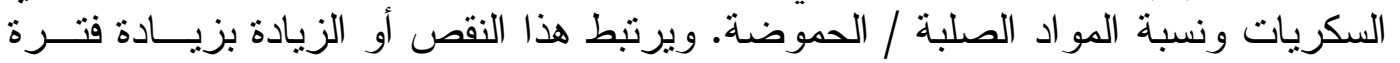

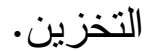

ץ- أدت التدفئة المتقطعة أثثاء التخزين إلي تقليل نسبة فقد الرطوبة و الثـــار التالفــة وتحســين

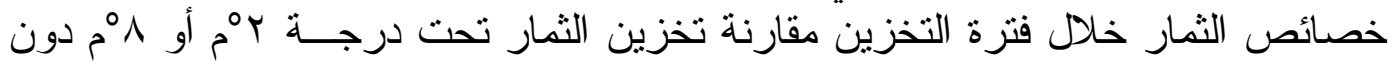

تدفئة.

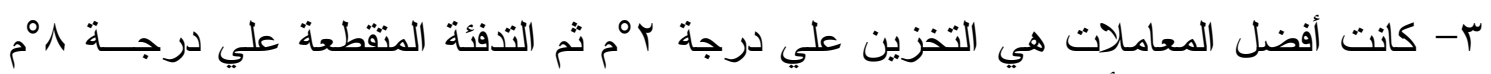
لمدة ^ ساعة كل أسبو عين.

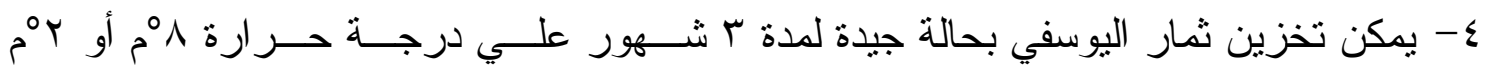

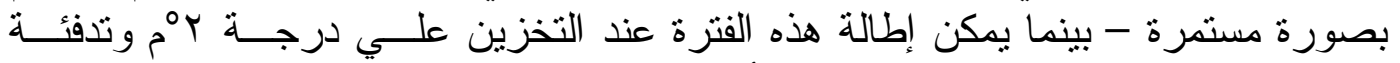

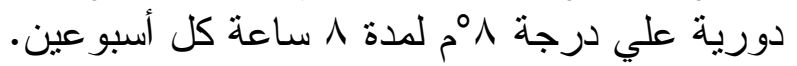

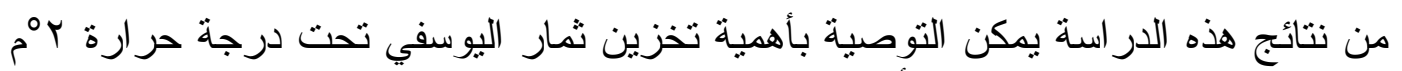

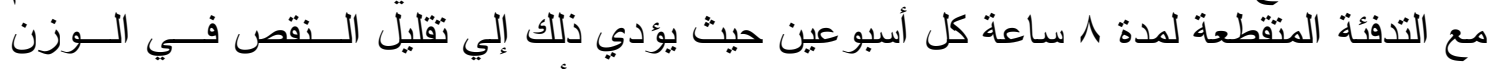
و الثمار التالفة بالإضافة إلي المحافظة علي خصائص الثمار أثناء فترة التخزين. 